\title{
Hadroproduction of open heavy flavour for PDF analyses
}

\section{Ilkka Helenius}

University of Jyvaskyla, Department of Physics, P.O. Box 35, FI-40014 University of Jyvaskyla, Finland

Helsinki Institute of Physics, P.O. Box 64, FI-00014 University of Helsinki, Finland

Institute for Theoretical Physics, Tübingen University, Auf der Morgenstelle 14, 72076

Tübingen, Germany

E-mail: ilkka.m.helenius@jyu.fi

\section{Hannu Paukkunen*}

University of Jyvaskyla, Department of Physics, P.O. Box 35, FI-40014 University of Jyvaskyla, Finland

Helsinki Institute of Physics, P.O. Box 64, FI-00014 University of Helsinki, Finland

E-mail: hannu.paukkunen@ jyu.fi

\begin{abstract}
Due to the large masses of the charm and bottom quarks, their production cross sections are calculable within the perturbative QCD. This makes the heavy-quark mesons important observables in high-energy collisions of protons and nuclei. However, the available calculations for heavyflavored-meson hadroproduction have been somewhat problematic in reliably describing the cross sections across the full kinematic range from zero to very high $p_{\mathrm{T}}$. This has put some question marks on the robustness of LHC heavy-flavored-meson measurements in studying the partonic structure of the colliding hadrons and nuclei. Here, we introduce SACOT- $m_{\mathrm{T}}$ - a novel scheme for open heavy-flavour hadroproduction within the general-mass variable-flavour-number formalism that solves this problem. The introduced scheme is an analogue of the SACOT- $\chi$ scheme often used for deeply-inelastic scattering in global analyses of PDFs.
\end{abstract}

XXVII International Workshop on Deep-Inelastic Scattering and Related Subjects - DIS2019 8-12 April, 2019

Torino, Italy

${ }^{*}$ Speaker. 


\section{Introduction}

The hadroproduction of open heavy flavour - D mesons in particular - has recently been advocated as a promising constraint for proton parton distribution functions (PDFs) [1, 2]. The theoretical description is typically based on the fixed flavour-number scheme (FFNS) [3], FONLL code [4], or FFNS matched to parton showers [5]. However, the use of e.g. FFNS calculation in conjunction with PDFs defined in variable flavour-number schemes (the commonly used general-purpose PDFs) may be too restrictive, and in this sense calculations within the framework of general-mass variable-flavour-number scheme (GM-VFNS) would be more natural. Here, we will discuss our novel implementation of the GM-VFNS, the so-called SACOT- $m_{\mathrm{T}}$ scheme [6].

\section{D-meson production in fixed flavour-number scheme}

Within FFNS - assuming no intrinsic heavy-quark content in the proton - the massive quarks $Q$ are always produced in pairs by the partonic processes, $g+g \rightarrow Q \bar{Q}+X, q+\bar{q} \rightarrow Q \bar{Q}+X, q+g \rightarrow$ $Q \bar{Q}+X$. The cross section for inclusive $Q$ production can be written as an integral of PDFs $f_{i}^{p}\left(x_{1}, \mu_{\text {fact }}^{2}\right)$ and partonic cross sections $d \hat{\sigma}^{i j}$,

$$
\frac{d \sigma(p+p \rightarrow Q+X)}{d p_{\mathrm{T}} d y}=\sum_{i j} \int d x_{1} d x_{2} f_{i}^{p}\left(x_{1}, \mu_{\mathrm{fact}}^{2}\right) \frac{d \hat{\sigma}^{i j \rightarrow Q+X}\left(\tau_{1}, \tau_{2}, m^{2}, \mu_{\mathrm{ren}}^{2}, \mu_{\text {fact }}^{2}\right)}{d p_{\mathrm{T}} d y} f_{j}^{p}\left(x_{2}, \mu_{\text {fact }}^{2}\right),
$$

where $y$ and $p_{\mathrm{T}}$ denote the rapidity and transverse momentum of the produced heavy quark. The factorization and renormalization scales are marked by $\mu_{\text {fact }}^{2}$ and $\mu_{\text {ren }}^{2}$. The kinematic variables $\tau_{1,2}$ are the "massive" Mandelstam variables,

$$
\tau_{1} \equiv p_{1} \cdot p_{3} / p_{1} \cdot p_{2}=m_{\mathrm{T}} e^{-y} /\left(\sqrt{s} x_{2}\right), \quad \tau_{2} \equiv p_{2} \cdot p_{3} / p_{1} \cdot p_{2}=m_{\mathrm{T}} e^{y} /\left(\sqrt{s} x_{1}\right), \quad m_{\mathrm{T}}^{2}=p_{\mathrm{T}}^{2}+m^{2},
$$

denoting the momenta of the incoming partons and outgoing heavy quark by $p_{1,2}$ and $p_{3}$, respectively. The partonic cross sections scale as $d \hat{\sigma}^{i j \rightarrow Q+X} \sim \tau_{1,2}^{-n}$, and, thanks to the heavy-quark mass, remain finite even at $p_{\mathrm{T}}=0$. The heavy-quark cross sections can be turned into, say $\mathrm{D}^{0}$-meson production cross sections by folding with $Q \rightarrow \mathrm{D}^{0}$ fragmentation functions (FFs) $D_{Q \rightarrow \mathrm{D}^{0}}(z)$. The fragmentation variable $z$ is not unique when the masses of the heavy quark and $\mathrm{D}^{0}$ meson are kept non zero. For simplicity, we define $z \equiv E_{\mathrm{D}^{0}} / E_{Q}$, where $E_{\mathrm{D}^{0}}$ and $E_{Q}$ are the energies of the $\mathrm{D}^{0}$ meson and heavy quark in the center-of-mass frame of the p-p collision. Assuming that the fragmentation is collinear we get,

$$
\frac{d \sigma\left(p+p \rightarrow \mathrm{D}^{0}+X\right)}{d P_{\mathrm{T}} d Y}=\sum_{i j} \int \frac{d z}{z} d x_{1} d x_{2} f_{i}^{p}\left(x_{1}, \mu_{\mathrm{fact}}^{2}\right) \frac{d \hat{\sigma}^{i j \rightarrow Q+X}}{d p_{\mathrm{T}} d y} f_{j}^{p}\left(x_{2}, \mu_{\mathrm{fact}}^{2}\right) D_{Q \rightarrow \mathrm{D}^{0}}(z),
$$

where the (lower case) partonic and (upper case) hadronic variables are related by

$$
\begin{aligned}
p_{\mathrm{T}}^{2} & =\frac{M_{\mathrm{T}}^{2} \cosh ^{2} Y-z^{2} m^{2}}{z^{2}}\left(1+\frac{M_{\mathrm{T}}^{2} \sinh ^{2} Y}{P_{\mathrm{T}}^{2}}\right)^{-1} \stackrel{P_{\mathrm{T}} \rightarrow \infty}{\longrightarrow}\left(\frac{P_{\mathrm{T}}}{z}\right)^{2}, \\
y & =\sinh ^{-1}\left(\frac{M_{\mathrm{T}} \sinh Y}{P_{\mathrm{T}}} \frac{p_{\mathrm{T}}}{m_{\mathrm{T}}}\right) \stackrel{P_{\mathrm{T}} \rightarrow \infty}{\longrightarrow} Y,
\end{aligned}
$$

and $M_{\mathrm{T}}=\sqrt{P_{\mathrm{T}}^{2}+M_{Q}^{2}}$ marks the hadronic transverse mass. While this framework appears to work well at low $P_{\mathrm{T}}$ (see e.g. [7]), the description seems to deteriorate towards high $P_{\mathrm{T}}$. Presumably, this can be attributed to the $\log \left(p_{\mathrm{T}}^{2} / \mathrm{m}^{2}\right)$ behaviour of the partonic cross sections which begin to dominate and should be resummed, as we will come to conlcude. 


\section{D-meson production in general-mass variable-flavour-number scheme}

The GM-VFNS description can be obtained from FFNS by resumming the $\log \left(p_{\mathrm{T}}^{2} / \mathrm{m}^{2}\right)$ terms that appear in FFNS. As an example, in Figure 1 the diagram (a) gives rise to such logarithmic behaviour when the initial-state gluon splits into a $Q \bar{Q}$ pair. This is only the first of the whole series of diagrams that are in GM-VFNS summed into the heavy-quark $\operatorname{PDF} f_{Q}^{p}$. Effectively, this

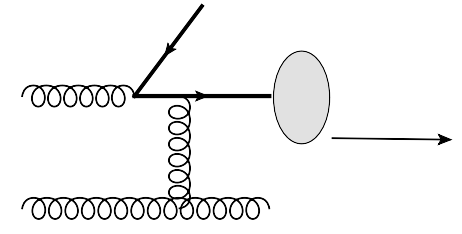

(a)

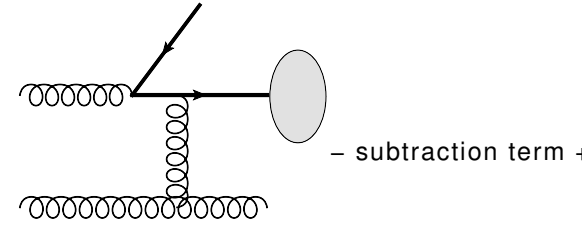

(a)

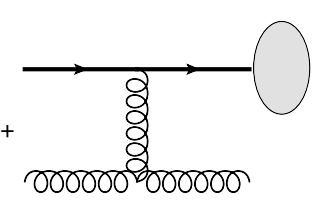

(c)

Figure 1: Origin of the heavy-quark initiated subprocess illustrated.

summation can be realized by including the heavy-quark initiated contribution (c) and a subtraction term (b) that avoids the double counting between diagrams (a) and (c). The contribution from $Q g \rightarrow Q+X$ channel, represented here by the diagram (c), can be written as

$$
\int \frac{d z}{z} d x_{1} d x_{2} f_{Q}^{p}\left(x_{1}, \mu_{\text {fact }}^{2}\right) \frac{d \hat{\sigma}^{Q g \rightarrow Q+X}\left(\tau_{1}, \tau_{2}\right)}{d p_{\mathrm{T}} d y} f_{g}^{p}\left(x_{2}, \mu_{\text {fact }}^{2}\right) D_{Q \rightarrow \mathrm{D}^{0}}(z)
$$

The subtraction term (b) is obtained from this same expression by replacing the heavy-quark PDF with its perturbative expression,

$$
f_{Q}^{p}\left(x, \mu_{\text {fact }}^{2}\right)=\left(\frac{\alpha_{s}}{2 \pi}\right) \log \left(\frac{\mu_{\text {fact }}^{2}}{m^{2}}\right) \int_{x}^{1} \frac{\mathrm{d} \ell}{\ell} P_{q g}\left(\frac{x}{\ell}\right) f_{g}^{p}\left(\ell, \mu_{\text {fact }}^{2}\right)+\mathscr{O}\left(\alpha_{s}^{2}\right),
$$

where $\alpha_{s}$ is the QCD coupling, and $P_{q g}$ is the usual gluon-to-quark splitting function. However, the exact form of $d \hat{\sigma}^{Q g \rightarrow Q+X}\left(\tau_{1}, \tau_{2}\right)$ in the above expressions is not unique [8]. In practice, we can only require that the zero-mass $\overline{\mathrm{MS}}$ expressions are recovered at high $p_{\mathrm{T}}$,

$$
\frac{d \hat{\sigma}^{Q g \rightarrow Q+X}\left(\tau_{1}, \tau_{2}\right)}{d p_{\mathrm{T}} d y} \stackrel{p_{\mathrm{T}} \rightarrow \infty}{\longrightarrow} \frac{d \hat{\sigma}^{q g \rightarrow q+X}\left(\tau_{1}^{0}, \tau_{2}^{0}\right)}{d p_{\mathrm{T}} d y}, \quad \tau_{1,2}^{0}=\tau_{1,2} \stackrel{m \rightarrow 0}{\longrightarrow} p_{\mathrm{T}} e^{\mp y} /\left(\sqrt{s} x_{2,1}\right) .
$$

The easiest option is to define $d \hat{\sigma}^{Q g \rightarrow Q+X}\left(\tau_{1}, \tau_{2}\right) \equiv d \hat{\sigma}^{q g \rightarrow q+X}\left(\tau_{1}^{0}, \tau_{2}^{0}\right)$, i.e. use the zero-mass expressions to begin with. This is known as the SACOT scheme [9]. The problem of this choice is that it leads to infinite cross sections towards $P_{\mathrm{T}} \rightarrow 0$ due to the $d \hat{\sigma}^{q g \rightarrow q+X} / d^{3} p \sim\left(\tau_{1,2}^{0}\right)^{-n}$ behaviour of the partonic cross sections. In the so-called FONLL scheme [4] this is avoided by multiplying the partonic cross section by an ad-hoc damping factor $p_{\mathrm{T}}^{2} /\left(p_{\mathrm{T}}^{2}+c^{2} m^{2}\right)$ with $c \sim 5$, which serves to tame the unphysical behaviour at small $p_{\mathrm{T}}$. Alternatively, the problematic behaviour can be avoided simply by retaining the kinematics of the $Q \bar{Q}$-pair production which, deep down, is the underlying process we describe. With this physical picture in mind, we define $d \hat{\sigma}^{Q g \rightarrow Q+X}\left(\tau_{1}, \tau_{2}\right) \equiv d \hat{\sigma}^{q g \rightarrow q+X}\left(\tau_{1}, \tau_{2}\right)$ taking $\tau_{1,2}=m_{\mathrm{T}} e^{\mp y} /\left(\sqrt{s} x_{2,1}\right)$ as in FFNS. This leads to well-behaved cross sections in the $P_{\mathrm{T}} \rightarrow 0$ limit. We call this the SACOT- $m_{\mathrm{T}}$ scheme, as it shares the same underlying idea as the SACOT- $\chi$ scheme in deeply-inelastic scattering [10].

Part of the $\log \left(p_{\mathrm{T}}^{2} / m^{2}\right)$ terms in FFNS come also from final-state splittings. As an example, Figure 2 shows a diagram in which an outgoing gluon splits into a $Q \bar{Q}$ pair. The result- 


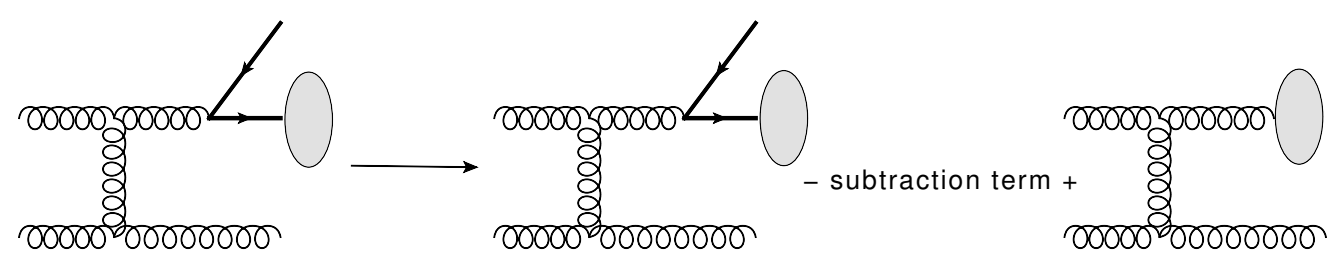

Figure 2: Origin of the gluon-fragmentation subprocess illustrated.

ing $\log \left(p_{\mathrm{T}}^{2} / \mathrm{m}^{2}\right)$ terms are absorbed into the fragmentation-scale $\left(\mu_{\mathrm{frag}}\right)$ dependent gluon FFs, $D_{g \rightarrow \mathrm{D}^{0}}\left(z, \mu_{\text {frag }}^{2}\right)$, giving rise to gluon-fragmentation contributions,

$$
\int \frac{d z}{z} d x_{1} d x_{2} f_{g}^{p}\left(x_{1}, \mu_{\text {fact }}^{2}\right) \frac{d \hat{\sigma}^{g g \rightarrow g+X}\left(\tau_{1}, \tau_{2}\right)}{d p_{\mathrm{T}} d y} f_{g}^{p}\left(x_{2}, \mu_{\text {fact }}^{2}\right) D_{g \rightarrow \mathrm{D}^{0}}\left(z, \mu_{\text {frag }}^{2}\right) .
$$

The subtraction term avoiding the double counting is again the same expression, but replacing the gluon FF by its perturbative form,

$$
D_{g \rightarrow \mathrm{D}^{0}}\left(x, \mu_{\text {frag }}^{2}\right)=\left(\frac{\alpha_{s}}{2 \pi}\right) \log \left(\frac{\mu_{\text {frag }}^{2}}{m^{2}}\right) \int_{x}^{1} \frac{\mathrm{d} \ell}{\ell} P_{q g}\left(\frac{x}{\ell}\right) D_{Q \rightarrow \mathrm{D}^{0}}(\ell)+\mathscr{O}\left(\alpha_{s}^{2}\right) .
$$

In line with our scheme choice, the $\overline{\mathrm{MS}}$ zero-mass matrix elements for $d \hat{\sigma}^{g g \rightarrow g+X}\left(\tau_{1}, \tau_{2}\right)$ with the massive expressions for $\tau_{1,2}$, are adopted. Even if the heavy quarks do not explicitly appear in the $g g \rightarrow g+X$ process, the underlying process is also here the $Q \bar{Q}$-pair production.

With this schematic justification, our "master formula" within GM-VFNS is,

$$
\begin{gathered}
\frac{d \sigma^{p p \rightarrow \mathrm{D}^{0}+X}}{d P_{\mathrm{T}} d Y}=\sum_{i j k} \int \frac{d z}{z} d x_{1} d x_{2} f_{i}^{p}\left(x_{1}, \mu_{\text {fact }}^{2}\right) \frac{d \hat{\sigma}^{i j \rightarrow k}\left(\tau_{1}, \tau_{2}, m, \mu_{\text {ren }}^{2}, \mu_{\text {fact }}^{2}, \mu_{\text {frag }}^{2}\right)}{d p_{\mathrm{T}} d y} \\
f_{j}^{p}\left(x_{2}, \mu_{\text {fact }}^{2}\right) D_{k \rightarrow \mathrm{D}^{0}}\left(z, \mu_{\text {frag }}^{2}\right) .
\end{gathered}
$$

Unlike in FFNS, all partonic subprocesses are included and the FFs are scale dependent. In the limit $p_{\mathrm{T}} \rightarrow 0$, the partonic cross sections reduce to FFNS, but towards $p_{\mathrm{T}} \rightarrow \infty$ they tend to the zero-mass $\overline{\mathrm{MS}}$ expressions. Our numerical realization of SACOT- $m_{\mathrm{T}}$ scheme is crafted around the Mangano-Nason-Ridolfi code [3] for heavy quarks, and the INCNLO code [11] for zero-mass partons. All terms up to $\mathscr{O}\left(\alpha_{s}^{3}\right)$ are included.

\section{Description of the $\mathrm{LHCb} \mathrm{D}^{0}$ data}

In Figure 3, we compare the LHCb $13 \mathrm{TeV} \mathrm{D}^{0}$ data [12] with our GM-VFNS theory calculation. The darker bands show the NNPDF3.1 (pch) [13] PDF uncertainty, and the lighter bands combine the scale-variation and PDF errors. We have used here the KKKS08 FFs [14]. The calculation agrees very well with the data though the scale variation leads to a significant uncertainty at small $P_{\mathrm{T}}$. We have found that, the contribution from the three FFNS processes, including the subtraction terms, is less than $10 \%$ for $P_{\mathrm{T}} \gtrsim 3 \mathrm{GeV}$ and only around $3 \%$ for $P_{\mathrm{T}} \gtrsim 10 \mathrm{GeV}$. This demonstrates that the $\log \left(p_{\mathrm{T}}^{2} / \mathrm{m}^{2}\right)$ terms in FFNS become quickly the dominant ones and must be resummed as done in GM-VFNS. A comparison using FFNS + parton-shower approach (with the same PDFs) is also presented. Here, we have used the POWHEG-Box event generator [5] matched to the Pythia 8 [15] parton shower. We see that the Powheg+Pythia setup has a tendency to 

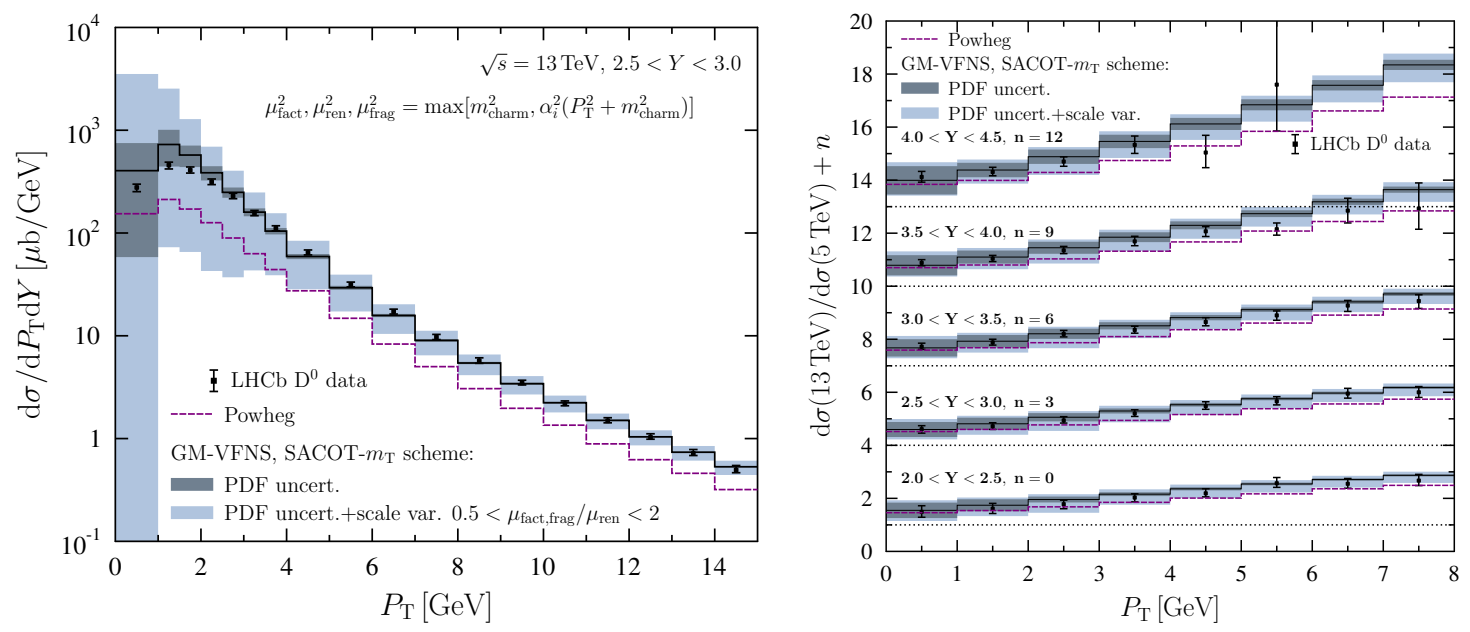

Figure 3: Left: LHCb $\mathrm{D}^{0}$ data [12] in p-p collisions at $\sqrt{s}=13 \mathrm{TeV}$ compared to our GM-VFNS calculation and the Powheg+Pythia framework. Right: Ratio between $\sqrt{s}=13 \mathrm{TeV}$ and $\sqrt{s}=5 \mathrm{TeV}$ data.

underpredict the experimental spectrum, and the ratio between two $\sqrt{s}$ is clearly flatter than the

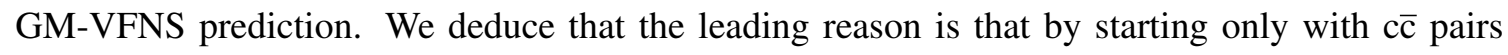
(generated by POWHEG) one neglects the contributions in which the parton shower excites the $c \bar{c}$ pair only later on. In GM-VFNS these are resummed to the scale-dependent FFs and, indeed, e.g. the gluon-to-D contributions can be around 50\% of the total budget. The gluon fragmentation also significantly changes the $x$ regions in which the PDFs are sampled. Thus, the use of FFNS-based computation when using D-meson data to fit GM-VFNS PDFs would inflict a potential bias.

\section{Acknowledgments}

The support by the Academy of Finland Projects 297058 \& 308301, the Carl Zeiss Foundation, and the state of Baden-Württemberg through bwHPC, are acknowledged. We have used the computing facilities of the Finnish IT Center for Science (CSC) in our work.

\section{References}

[1] O. Zenaiev et al., Eur. Phys. J. C 75 (2015) 396.

[2] R. Gauld and J. Rojo, Phys. Rev. Lett. 118 (2017) 072001.

[3] M. L. Mangano, et al., Nucl. Phys. B 373 (1992) 295.

[4] M. Cacciari, et al., JHEP 9805 (1998) 007.

[5] S. Frixione, et al., JHEP 0709 (2007) 126.

[6] I. Helenius and H. Paukkunen, JHEP 1805 (2018) 196.

[7] O. Zenaiev, Eur. Phys. J. C 77 (2017) no.3, 151.

[8] R. S. Thorne and W. K. Tung, arXiv:0809.0714 [hep-ph].

[9] B. A. Kniehl et al., Phys. Rev. D 71 (2005) 014018.

[10] M. Guzzi, et al., Phys. Rev. D 86 (2012) 053005.

[11] F. Aversa, et al., Nucl. Phys. B 327 (1989) 105.

[12] R. Aaij et al., JHEP 1603 (2016) 159.

[13] R. D. Ball et al., Eur. Phys. J. C 77 (2017) 663.

[14] T. Kneesch, et al., Nucl. Phys. B 799 (2008) 34.

[15] T. Sjöstrand et al., Comput. Phys. Commun. 191 (2015) 159. 